\title{
Research Article \\ On System of Generalized Vector Quasiequilibrium Problems with Applications
}

\author{
Jian-Wen Peng ${ }^{1}$ and Lun Wan ${ }^{2}$ \\ ${ }^{1}$ College of Mathematics and Computer Science, Chongqing Normal University, Chongqing 400047, China \\ ${ }^{2}$ Scientific Research Office, Chongqing Normal University, Chongqing 400047, China
}

Correspondence should be addressed to Jian-Wen Peng, jwpeng6@yahoo.com.cn

Received 3 July 2009; Accepted 19 August 2009

Recommended by Marco Squassina

\begin{abstract}
We introduce a new system of generalized vector quasiequilibrium problems which includes system of vector quasiequilibrium problems, system of vector equilibrium problems, and vector equilibrium problems, and so forth in literature as special cases. We prove the existence of solutions for this system of generalized vector quasi-equilibrium problems. Consequently, we derive some existence results of a solution for the system of generalized quasi-equilibrium problems and the generalized Debreu-type equilibrium problem for both vector-valued functions and scalar-valued functions.
\end{abstract}

Copyright (C) 2009 J.-W. Peng and L. Wan. This is an open access article distributed under the Creative Commons Attribution License, which permits unrestricted use, distribution, and reproduction in any medium, provided the original work is properly cited.

\section{Introduction and Formulations}

In the resent years, the vector equilibrium problems have been studied in [1-7] and the references therein which is a unified model of several problems, for instance, vector variational inequality, vector variational-like inequality, vector complementarity problems, vector optimization problems. A comprehensive bibliography on vector equilibrium problems, vector variational inequalities, vector variational-like inequalities and their generalizations can be found in a recent volume [1]. Ansari and Yao [8] and Chiang et al. [9] introduced and studied some vector quasi-equilibrium problems which generalized those quasi-equilibrium problems in [10-17] to the case of vector-valued function. Very recently, the system of vector equilibrium problems was introduced by Ansari et al. [18] with applications in Nash-type equilibrium problem for vector-valued functions. The system of vector quasi-equilibrium problems was introduced by Ansari et al. [19] with applications in Debreu-type equilibrium problem for vector-valued functions. As a generalization of the above models, we introduce a new system of generalized vector quasi-equilibrium problems, that is, a family of generalized quasi-equilibrium problems for vector-valued maps defined on a product set. 
Throughout this paper, for a set $A$ in a topological space, we denote by co $A$, int $A$, $\overline{\mathrm{CO}} A$ the convex hull, interior, and the convex closure of $A$, respectively.

Let $I$ be an index set. For each $i \in I$, let $Z_{i}, E_{i}$ and let $F_{i}$ be topological vector spaces. Consider two family of nonempty convex subsets $\left\{X_{i}\right\}_{i \in I}$ with $X_{i} \subseteq E_{i}$ and $\left\{Y_{i}\right\}_{i \in I}$ with $Y_{i} \subseteq F_{i}$. Let

$$
E=\prod_{i \in I} E_{i}, \quad X=\prod_{i \in I} X_{i}, \quad F=\prod_{i \in I} F_{i}, \quad Y=\prod_{i \in I} Y_{i}
$$

An element of the set $X^{i}=\prod_{j \in I \backslash i} X_{i}$ will be denoted by $x^{i}$, therefore, $x \in X$ will be written as $x=\left(x^{i}, x_{i}\right) \in X^{i} \times X_{i}$. Similarly, an element of the set $Y$ will be denoted by $y=\left(y^{i}, y_{i}\right) \in Y^{i} \times Y_{i}$. For each $i \in I$, let $C_{i}: X \rightarrow 2^{Z_{i}}, D_{i}: X \times Y \rightarrow 2^{X_{i}}$ and $T_{i}: X \times Y \rightarrow 2^{Y_{i}}$ be set-valued maps with nonempty values, and let $f_{i}: X \times Y \times X_{i} \rightarrow Z_{i}$ be a the vector-valued function. Then the system of generalized vector quasi-equilibrium problems (in Short, SGVQEP) is to find $(\bar{x}, \bar{y})=\left(\bar{x}^{i}, \bar{x}_{i}, \bar{y}^{i}, \bar{y}_{i}\right)$ in $X \times Y$ such that for each $i \in I$,

$$
\bar{x}_{i} \in D_{i}(\bar{x}, \bar{y}), \quad \bar{y}_{i} \in T_{i}(\bar{x}, \bar{y}): f_{i}\left(\bar{x}, \bar{y}, z_{i}\right) \notin-\operatorname{int} C_{i}(\bar{x}), \quad \forall z_{i} \in D_{i}(\bar{x}, \bar{y})
$$

Here are some special cases of the (SGVQEP).

(i) For each $i \in I$, let $\phi_{i}: X \times Y \rightarrow Z_{i}$ be a vector-valued function. We define a trifunction $f_{i}: X \times Y \times X_{i} \rightarrow Z_{i}$ as $f_{i}\left(x, y, u_{i}\right)=\phi_{i}\left(x^{i}, y, u_{i}\right)-\phi_{i}(x, y), \forall\left(x, y, u_{i}\right) \in X \times Y \times X_{i}$. Then the (SGVQEP) reduces to the generalized Debreu-type equilibrium problem for vectorvalued functions (in short, G-Debreu VEP), which is to find $(\bar{x}, \bar{y})=\left(\bar{x}^{i}, \bar{x}_{i}, \bar{y}^{i}, \bar{y}_{i}\right)$ in $X \times Y$ such that for each $i \in I$,

$$
\bar{x}_{i} \in D_{i}(\bar{x}, \bar{y}), \quad \bar{y}_{i} \in T_{i}(\bar{x}, \bar{y}): \phi_{i}\left(\bar{x}^{i}, \bar{y}, z_{i}\right)-\phi_{i}(\bar{x}, \bar{y}) \notin-\operatorname{int} C_{i}(\bar{x}), \quad \forall z_{i} \in D_{i}(\bar{x}, \bar{y}) .
$$

(ii) We denote by $R$ and $R^{+}$the set of real numbers and the set of real nonnegative numbers, respectively. For each $i \in I$, if $Z_{i}=R$, and $C_{i}(x)=R^{+}$for all $x \in X$, then the (SGVQEP) reduces to the system of generalized quasi-equilibrium problems (in short, SGQEP), which is to find $(\bar{x}, \bar{y})=\left(\bar{x}^{i}, \bar{x}_{i}, \bar{y}^{i}, \bar{y}_{i}\right)$ in $X \times Y$ such that for each $i \in I$,

$$
\bar{x}_{i} \in D_{i}(\bar{x}, \bar{y}), \quad \bar{y}_{i} \in T_{i}(\bar{x}, \bar{y}): f_{i}\left(\bar{x}, \bar{y}, z_{i}\right) \geq 0, \quad \forall z_{i} \in D_{i}(\bar{x}, \bar{y})
$$

And the G-Debreu VEP reduces to the generalized Debreu-type equilibrium problem for scalar-valued functions (in short, G-Debreu EP), which is to find $(\bar{x}, \bar{y})=\left(\bar{x}^{i}, \bar{x}_{i}, \bar{y}^{i}, \bar{y}_{i}\right)$ in $X \times Y$ such that for each $i \in I$,

$$
\bar{x}_{i} \in D_{i}(\bar{x}, \bar{y}), \quad \bar{y}_{i} \in T_{i}(\bar{x}, \bar{y}): \phi_{i}\left(\bar{x}^{i}, \bar{y}, z_{i}\right) \geq \phi_{i}(\bar{x}, \bar{y}), \quad \forall z_{i} \in D_{i}(\bar{x}, \bar{y}) .
$$

(iii) Let $Y=\{\bar{y}\}$. For each $i \in I$, let $D_{i}(x, \bar{y})=A_{i}(x), T_{i}(x, \bar{y})=\left\{\bar{y}_{i}\right\}$ for all $x \in X$, where $A_{i}: X \rightarrow 2^{X_{i}}$ is a set-valued map. We define a function $\varphi_{i}: X \times X_{i} \rightarrow Z_{i}$ and a function $h_{i}: X \times Y \rightarrow Z_{i}$ as $\varphi_{i}\left(x, z_{i}\right)=f_{i}\left(x, \bar{y}, z_{i}\right)$, for all $\left(x, z_{i}\right) \in X \times X_{i}$, and $h_{i}(x)=\phi_{i}(x, \bar{y})$, for all $x \in X$, then (SGVQEP) and (G-Debreu VEP), respectively, reduce to the system of vector quasi-equilibrium problems and the (Debreu VEP) introduced by Ansari 
et al. [19] which contain those mathematical in $[18,20]$ as special cases. The (SGQEP) reduces to the mathematical models in [21, page 286] and [22, pages 152-153] and the (G-Debreu EP) reduces to the abstract economy in [23, page 345] which contains the noncooperative game in [24] as a special case.

(iv) If the index set $I$ is singleton, $D(x, y)=D_{i}(x), T(x, y)=T(x)$, and $C(x)=C$, then the (SGVQEP) becomes the implicit vector variational inequality in [9] and the (SGQEP) reduces to the quasi-equilibrium problem investigated in [14-17].

The rest of this paper is arranged in the following manner. The following section deals with some preliminary definitions, notations and results which will be used in the sequel. In Section 3, we establish existence results for a solution to the (SGVQEP) and the (SGQEP) with or without involving $\Phi$-condensing maps by using similar techniques in [19]. In Section 4, as applications of the results of Section 3, we derive some existence results of a solution for the (G-Debreu VEP) and the the (G-Debreu EP).

\section{Preliminaries}

In order to prove the main results, we need the following definitions.

Definition $2.1([19,25])$. Let $M$ be a nonempty convex subset of a topological vector space $E$ and $Z$ a real topological space with a closed and convex cone $P$ with apex at the origin. A vector-valued function $\varphi: M \rightarrow Z$ is called

(i) $P$-quasifunction if and only if, for all $z \in Z$, the set $\{x \in M: \varphi(x) \in z-P\}$ is convex,

(ii) natural $P$-quasifunction if and only if, $\forall x, y \in M$, and $\lambda \in[0,1], \varphi(\lambda x+(1-\lambda) y) \in$ $\operatorname{co}\{\varphi(x), \varphi(y)\}-P$.

Definition 2.2 ([13]). Let $X$ and $Y$ be two topological spaces. $T: X \rightarrow 2^{Y}$ be a set-valued map. Then $T$ is said to be upper semicontinuous if the set $\{x \in X: T(x) \subseteq V\}$ is open in $X$ for every open subset $V$ of $Y$. Also $T$ is said to be lower semicontinuous if the set $\{x \in X: T(x) \cap V\}$ is open in $X$ for every open subset $V$ of $Y \cdot T$ is said to have open lower sections if the set $T^{-1}(y)=\{x \in X: y \in T(x)\}$ is open in $X$ for each $y \in Y$.

Definition 2.3 ([26]). Let $E$ be a Hausdorff topological space and $L$ a lattice with least element, denoted by 0 . A map $\Phi: 2^{E} \rightarrow L$ is a measure of noncompactness provided that the following conditions hold $\forall M, N \in 2^{E}$ :

(i) $\Phi(M)=0$ iff $M$ is precompact (i.e., it is relatively compact),

(ii) $\Phi(\overline{\mathrm{co}} M)=\Phi(M)$,

(iii) $\Phi(M \cup N)=\max \{\Phi(M), \Phi(N)\}$.

Definition 2.4 ([26]). Let $\Phi: 2^{E} \rightarrow L$ be a measure of noncompactness on $E$ and $X \subseteq E$. A set-valued map $T: X \rightarrow 2^{E}$ is called $\Phi$-condensing provided that, if $M \subseteq X$ with $\Phi(T(M)) \geq$ $\Phi(M)$, then $M$ is relatively compact.

Remark 2.5. Note that every set-valued map defined on a compact set is $\Phi$-condensing for any measure of noncompactness $\Phi$. If $E$ is locally convex and $T: X \rightarrow 2^{E}$ is a compact set-valued map (i.e., $T(X)$ is precompact), then $T$ is $\Phi$-condensing for any measure of noncompactness $\Phi$. It is clear that if $T: X \rightarrow 2^{E}$ is $\Phi$-condensing and $T^{*}: X \rightarrow 2^{E}$ satisfies $T^{*}(x) \subseteq T(x) \forall x \in$ $X$, then $T^{*}$ is also $\Phi$-condensing. 
We will use the following particular forms of two maximal element theorems for a family of set-valued maps due to Deguire et al. [27, Theorem 7] and Chebbi and Florenzano [28, Corollary 4].

Lemma 2.6 $([19,27])$. Let $\left\{X_{i}\right\}_{i \in I}$ be a family of nonempty convex subsets where each $X_{i}$ is contained in a Hausdorff topological vector space $E_{i}$, For each $i \in I$, let $S_{i}: X \rightarrow 2^{X_{i}}$ be a set-valued map such that

(i) for each $i \in I, S_{i}(x)$ is convex,

(ii) for each $x \in X, x_{i} \notin S_{i}(x)$,

(iii) for each $y_{i} \in X_{i}, S_{i}^{-1}\left(y_{i}\right)$ is open in $X$.

(iv) there exist a nonempty compact subset $N$ of $X$ and a nonempty compact convex subset $B_{i}$ of $X_{i}$ for each $i \in I$ such that for each $x \in X \backslash N$ there exists $i \in I$ satisfying $S_{i}(x) \cap B_{i} \neq \emptyset$. Then there exists $\bar{x} \in X$ such that $S_{i}(\bar{x})=\emptyset$ for all $i \in I$.

Lemma $2.7([19,28])$. Let I be any index set and $\left\{X_{i}\right\}_{i \in I}$ be a family of nonempty, closed and convex subsets where each $X_{i}$ is contained in a locally convex Hausdorff topological vector space $E_{i}$. For each $i \in I$, let $S_{i}: X \rightarrow 2^{X_{i}}$ be a set-valued map. Assume that the set-valued map $S: X \rightarrow 2^{X}$ defined as $S(x)=\prod_{i \in I} S_{i}(x), \forall x \in X$, is $\Phi$-condensing and the conditions (i), (ii), (iii) of Lemma 2.6 hold. Then there exists $\bar{x} \in X$ such that $S_{i}(\bar{x})=\emptyset$ for all $i \in I$.

\section{Existence Results}

An existence result of a solution for the system of generalized vector quasi-equilibrium problems with or without $\Phi$-condensing maps are will shown in this section.

Theorem 3.1. Let $I$ be any index set. For each $i \in I$, let $Z_{i}$ be a topological vector space, let $E_{i}$ and $F_{i}$ be two Hausdorff topological vector spaces, let $X_{i} \subseteq E_{i}$ and $Y_{i} \subseteq F_{i}$ be nonempty and convex subsets, let $D_{i}: X \times Y \rightarrow 2^{X_{i}}$ and $T_{i}: X \times Y \rightarrow 2^{Y_{i}}$ be set-valued maps with nonempty convex values and open lower sections, and the set $W_{i}=\left\{(x, y) \in X \times Y: x_{i} \in D_{i}(x, y)\right.$ and $\left.y_{i} \in T_{i}(x, y)\right\}$ be closed in $X \times Y$ and let $f_{i}: X \times Y \times X_{i} \rightarrow Z_{i}$ be a vector-valued function. For each $i \in I$, let $C_{i}: X \rightarrow 2^{Z_{i}}$ be a set-valued map such that $C_{i}(x)$ be a proper closed and convex cone with apex at the origin and $\operatorname{int} C_{i}(x) \neq \emptyset$ for all $x \in X$ and $P_{i}=\cap_{x \in X} C_{i}(x)$. Assume that

(i) for all $x=\left(x^{i}, x_{i}\right) \in X$, for all $y \in Y, f_{i}\left(x, y, x_{i}\right) \notin-\operatorname{int} C_{i}(x)$;

(ii) for each $(x, y) \in X \times Y, z_{i} \mapsto f_{i}\left(x, y, z_{i}\right)$ is natural $P_{i}$-quasifunction;

(iii) for all $z_{i} \in X_{i}$, the set $\left\{(x, y) \in X \times Y: f_{i}\left(x, y, z_{i}\right) \notin-\operatorname{int} C_{i}(x)\right\}$ is closed in $X \times Y$;

(iv) there exist nonempty and compact subsets $N \subseteq X$ and $K \subseteq Y$ and nonempty, compact and convex subsets $B_{i} \subseteq X_{i}, A_{i} \subseteq Y_{i}$ for each $i \in I$ such that $\forall(x, y)=\left(x^{i}, x_{i}, y\right) \in$ $X \times Y \backslash N \times K \exists i \in I$ and $\exists \bar{u}_{i} \in B_{i}, \bar{v}_{i} \in A_{i}$ satisfying $\bar{u}_{i} \in D_{i}(x, y), \bar{v}_{i} \in T_{i}(x, y)$ and $f_{i}\left(x, y, \bar{u}_{i}\right) \in-\operatorname{int} C_{i}(x)$.

Then, there exists $(\bar{x}, \bar{y})=\left(\bar{x}^{i}, \bar{x}_{i}, \bar{y}^{i}, \bar{y}_{i}\right)$ in $X \times Y$ such that for each $i \in I$,

$$
\bar{x}_{i} \in D_{i}(\bar{x}, \bar{y}), \quad \bar{y}_{i} \in T_{i}(\bar{x}, \bar{y}): f_{i}\left(\bar{x}, \bar{y}, z_{i}\right) \notin-\operatorname{int} C_{i}(\bar{x}), \quad \forall z_{i} \in D_{i}(\bar{x}, \bar{y}) .
$$

That is, the solution set of the (SGVQEP) is nonempty. 
Proof. For each $i \in I$, let us define a set-valued map $Q_{i}: X \times Y \rightarrow 2^{X_{i}}$ by

$$
Q_{i}(x, y)=\left\{z_{i} \in X_{i}: f_{i}\left(x, y, z_{i}\right) \in-\operatorname{int} C_{i}(x)\right\}, \quad \forall(x, y) \in X \times Y
$$

Then, $\forall i \in I$ and $\forall(x, y) \in X \times Y, Q_{i}(x, y)$ is a convex set.

To prove it, let us fix arbitrary $i \in I$ and $(x, y) \in X \times Y$. Let $z_{i 1}, z_{i 2} \in Q_{i}(x, y)$ and $\lambda \in[0,1]$, then we have

$$
f_{i}\left(x, y, z_{i_{j}}\right) \in-\operatorname{int} C_{i}(x), \quad \text { for } j=1,2
$$

Since $f_{i}(x, y, \cdot)$ is natural $P_{i}$-quasifunction, $\exists \mu \in[0,1]$ such that

$$
f_{i}\left(x, y, \lambda z_{i_{1}}+(1-\lambda) z_{i_{2}}\right) \in \mu f_{i}\left(x, y, z_{i_{1}}\right)+(1-\mu) f_{i}\left(x, y, z_{i_{2}}\right)-P_{i} .
$$

From (3.3) and (3.4), we get

$$
f_{i}\left(x, y, \lambda z_{i_{1}}+(1-\lambda) z_{i_{2}}\right) \in-\operatorname{int} C_{i}(x)-\operatorname{int} C_{i}(x)-P_{i} \subseteq-\operatorname{int} C_{i}(x) .
$$

Hence $\lambda z_{i_{1}}+(1-\lambda) z_{i_{2}} \in Q_{i}(x, y)$ and, therefore, $Q_{i}(x, y)$ is convex.

It follows from condition (i) that, for each $i \in I$ and for all $(x, y)=\left(x^{i}, x_{i}, y\right) \in X \times Y$,

$$
x_{i} \notin Q_{i}(x, y) .
$$

It follows from condition (iii) that for each $i \in I$ and each $z_{i} \in X_{i}$, the set

$$
Q_{i}^{-1}\left(z_{i}\right)=\left\{(x, y) \in X \times Y: f_{i}\left(x, y, z_{i}\right) \in-\operatorname{int} C_{i}(x)\right\}
$$

is open in $X_{i}$. That is, $Q_{i}$ has open lower sections on $X \times Y$. For each $i \in I$, we also define another set-valued map $S_{i}: X \times Y \rightarrow 2^{X_{i} \times Y_{i}}$ by

$$
S_{i}(x, y)= \begin{cases}{\left[D_{i}(x, y) \cap Q_{i}(x, y)\right] \times T_{i}(x, y),} & \text { if }(x, y) \in W_{i} \\ D_{i}(x, y) \times T_{i}(x, y), & \text { if }(x, y) \notin W_{i}\end{cases}
$$

Then, it is clear that $\forall i \in I$ and $\forall(x, y) \in X \times Y, S_{i}(x, y)$ is convex, and $\left(x_{i}, y_{i}\right) \notin S_{i}(x, y)$. Since $\forall i \in I$ and $\forall\left(u_{i}, v_{i}\right) \in X_{i} \times Y_{i}$,

$$
\begin{aligned}
S_{i}^{-1}\left(u_{i}, v_{i}\right)= & {\left[Q_{i}^{-1}\left(u_{i}\right) \cap\left(D_{i}^{-1}\left(u_{i}\right)\right) \cap\left(T_{i}^{-1}\left(v_{i}\right)\right)\right] } \\
& \cup\left[\left(X \times Y \backslash W_{i}\right) \cap\left(D_{i}^{-1}\left(u_{i}\right)\right) \cap\left(T_{i}^{-1}\left(v_{i}\right)\right)\right],
\end{aligned}
$$

and $D_{i}^{-1}\left(u_{i}\right), T_{i}^{-1}\left(v_{i}\right), Q_{i}^{-1}\left(u_{i}\right)$, and $X \times Y \backslash W_{i}$ are open in $X \times Y$, we have $S_{i}^{-1}\left(u_{i}, v_{i}\right)$ is open in $X \times Y$. 
From condition (iv), there exist a nonempty and compact subset $N \times K \subseteq X \times Y$ and a nonempty, compact, and convex subset $B_{i} \times A_{i} \subseteq X_{i} \times Y_{i}$ for each $i \in I$ such that $\forall(x, y)=$ $\left(x^{i}, x_{i}, y\right) \in X \times Y \backslash N \times K \exists i \in I$ and $\exists\left(\bar{u}_{i}, \bar{v}_{i}\right) \in S_{i}(x, y) \cap\left(B_{i} \times A_{i}\right)$. Hence, by Lemma 2.6, $\exists(\bar{x}, \bar{y}) \in X \times Y$ such that $S_{i}(\bar{x}, \bar{y})=\emptyset, \forall i \in I$. Since $\forall i \in I$ and $\forall(x, y) \in X \times X, D_{i}(x, y)$ and $T_{i}(x, y)$ are nonempty, we have $(\bar{x}, \bar{y}) \in W_{i}$ and $D_{i}(\bar{x}, \bar{y}) \cap Q_{i}(\bar{x}, \bar{y})=\emptyset, \forall i \in I$. This implies $(\bar{x}, \bar{y}) \in W_{i}$ and $D_{i}(\bar{x}, \bar{y}) \cap Q_{i}(\bar{x}, \bar{y})=\emptyset, \forall i \in I$. Therefore, $\forall i \in I$,

$$
\bar{x}_{i} \in D_{i}(\bar{x}, \bar{y}), \quad \bar{y}_{i} \in T_{i}(\bar{x}, \bar{y}), \quad f_{i}\left(\bar{x}, \bar{y}, z_{i}\right) \notin-\operatorname{int} C_{i}(\bar{x}), \quad \forall z_{i} \in D_{i}(\bar{x}, \bar{y})
$$

That is, the solution set of the (SGVQEP) is nonempty.

Remark 3.2. (1) The condition (iii) of Theorem 3.1 is satisfied if the following conditions hold $\forall i \in I$ :

(a) $C_{i}: X \rightarrow 2^{Z_{i}}$ is a set-valued map such that $\operatorname{int} C_{i}(x) \neq \emptyset$ for each $x \in X$ and the set-valued map $M_{i}=Z_{i} \backslash\left(-\operatorname{int} C_{i}\right): X \rightarrow 2^{Z_{i}}$ is upper semicontinuous;

(b) for all $z_{i} \in X_{i}$, the map $(x, y) \mapsto f_{i}\left(x, y, z_{i}\right)$ is continuous on $X \times Y$;

(2) If $\forall i \in I$, and $\forall x \in X, C_{i}(x)=C_{i}$, a (fixed) proper, closed and convex cone in $Z_{i}$, then the condition (ii) and (iii) of Theorem 3.1 can be replaced, respectively, by the following conditions:

(c) $\forall i \in I$, the vector-valued function $\forall(x, y) \in X \times Y, z_{i} \mapsto f_{i}\left(x, y, z_{i}\right)$ is $C_{i^{-}}$ quasifunction;

(d) $\forall i \in I, \forall z_{i} \in X_{i}$, the map $(x, y) \mapsto f_{i}\left(x, y, z_{i}\right)$ is $C_{i}$-upper semicontinuous on $X \times Y$;

(3) Theorem 3.1 extends and generalizes in [19, Theorem 2], [20, Theorem 2.1] and $[18$, Theorem 2.1$]$ in several ways.

(4) If $\forall i \in I, X_{i}$ is a nonempty, compact and convex subset of a Hausdorff topological vector space $E_{i}$, then the conclusion of Theorem 3.1 holds without condition (iv).

Theorem 3.3. Let $I$ be any index set. For each $i \in I$, let $Z_{i}$ be a topological vector space, let $E_{i}$ and $F_{i}$ be two locally convex Hausdorff topological vector spaces, let $X_{i} \subseteq E_{i}$ and $Y_{i} \subseteq F_{i}$ be nonempty, closed and convex subsets, let $D_{i}: X \times Y \rightarrow 2^{X_{i}}$ and $T_{i}: X \times Y \rightarrow 2^{Y_{i}}$ be set-valued maps with nonempty convex values and open lower sections, the set $W_{i}=\left\{(x, y) \in X \times Y: x_{i} \in D_{i}(x, y)\right.$ and $\left.y_{i} \in T_{i}(x, y)\right\}$ be closed in $X \times Y$ and $f_{i}: X \times Y \times X_{i} \rightarrow Z_{i}$ be a vector-valued function. For each $i \in I$, let $C_{i}: X \rightarrow 2^{Z_{i}}$ be a set-valued map such that $C_{i}(x)$ be a proper closed and convex cone with apex at the origin and int $C_{i}(x) \neq \emptyset$ for all $x \in X$ and $P_{i}=\cap_{x \in X} C_{i}(x)$. Assume that the set-valued map $D \times T=\left(\prod_{i \in I} D_{i} \times \prod_{i \in I} T_{i}\right): X \times Y \rightarrow 2^{X \times Y}$ defined as $(D \times T)(x, y)=\prod_{i \in I} D_{i}(x, y) \times \prod_{i \in I} T_{i}(x, y)$, $\forall(x, y) \in X \times Y$, is $\Phi$-condensing and for each $i \in I$, the conditions (i), (ii) and (iii) of Theorem 3.1 hold. Then the solution set of the (SGVQEP) is nonempty.

Proof. In view of Lemma 2.7 and the proof of Theorem 3.1, it is sufficient to show that the set-valued map $S: X \times Y \rightarrow 2^{X \times Y}$ defined as $S(x, y)=\prod_{i \in I} S_{i}(x, y)$, for all $(x, y) \in X \times Y$, is $\Phi$-condensing, where $S_{i}$ 's are the same as in the proof of Theorem 3.1. By the definition of $S_{i}, S_{i}(x, y) \subseteq D_{i}(x, y) \times T_{i}(x, y)$ for all $(x, y) \in X \times Y$ and for each $i \in I$, and therefore $S(x, y) \subseteq D(x, y) \times T(x, y)$ for all $(x, y) \in X \times Y$. Since $D \times T$ is $\Phi$-condensing, by Remark 2.5, we have $S$ is also $\Phi$-condensing. 
By Theorem 3.1 and Remark 3.2, we can easily get the following result.

Corollary 3.4. Let $I$ be any index set. For each $i \in I$, let $E_{i}$ and $F_{i}$ be two Hausdorff topological vector spaces, let $X_{i} \subseteq E_{i}$ and $Y_{i} \subseteq F_{i}$ be nonempty and convex subsets, let $D_{i}: X \times Y \rightarrow 2^{X_{i}}$ and $T_{i}: X \times Y \rightarrow 2^{Y_{i}}$ be set-valued maps with nonempty convex values and open lower sections, let the set $W_{i}=\left\{(x, y) \in X \times Y: x_{i} \in D_{i}(x, y)\right.$ and $\left.y_{i} \in T_{i}(x, y)\right\}$ be closed in $X \times Y$, and $f_{i}: X \times Y \times X_{i} \rightarrow R$ be a function. Assume that

(i) for all $x=\left(x^{i}, x_{i}\right) \in X$, for all $y \in Y, f_{i}\left(x, y, x_{i}\right) \geq 0$;

(ii) for each $(x, y) \in X \times Y, z_{i} \mapsto f_{i}\left(x, y, z_{i}\right)$ is quasiconvex;

(iii) for all $z_{i} \in X_{i}$, the set $\left\{(x, y) \in X \times Y: f_{i}\left(x, y, z_{i}\right) \geq 0\right\}$ is closed in $X \times Y$;

(iv) there exist nonempty and compact subsets $N \subseteq X$ and $K \subseteq Y$ and nonempty, compact and convex subsets $B_{i} \subseteq X_{i}, A_{i} \subseteq Y_{i}$ for each $i \in I$ such that $\forall(x, y)=\left(x^{i}, x_{i}, y\right) \in$ $X \times Y \backslash N \times K \exists i \in I$ and $\exists \bar{u}_{i} \in B_{i}, \bar{v}_{i} \in A_{i}$ satisfying $\bar{u}_{i} \in D_{i}(x, y), \bar{v}_{i} \in T_{i}(x, y)$ and $f_{i}\left(x, y, \bar{u}_{i}\right)<0$.

Then, there exists $(\bar{x}, \bar{y})=\left(\bar{x}^{i}, \bar{x}_{i}, \bar{y}^{i}, \bar{y}_{i}\right)$ in $X \times Y$ such that for each $i \in I$,

$$
\bar{x}_{i} \in D_{i}(\bar{x}, \bar{y}), \quad \bar{y}_{i} \in T_{i}(\bar{x}, \bar{y}): f_{i}\left(\bar{x}, \bar{y}, z_{i}\right) \geq 0, \quad \forall z_{i} \in D_{i}(\bar{x}, \bar{y})
$$

That is, the solution set of the (SGQEP) is nonempty.

By Theorem 3.3, we can easily get the following result.

Corollary 3.5. Let $I$ be any index set. For each $i \in I$, let $Z_{i}$ be a topological vector space, let $E_{i}$ and $F_{i}$ be two locally convex Hausdorff topological vector spaces, let $X_{i} \subseteq E_{i}$ and $Y_{i} \subseteq F_{i}$ be nonempty, closed and convex subsets, let $D_{i}: X \times Y \rightarrow 2^{X_{i}}$ and $T_{i}: X \times Y \rightarrow 2^{Y_{i}}$ be set-valued maps with nonempty convex values and open lower sections, the set $W_{i}=\left\{(x, y) \in X \times Y: x_{i} \in D_{i}(x, y)\right.$ and $y_{i} \in$ $\left.T_{i}(x, y)\right\}$ be closed in $X \times Y$ and $f_{i}: X \times Y \times X_{i} \rightarrow R$ be a function. Assume that the set-valued map $D \times T=\left(\prod_{i \in I} D_{i} \times \prod_{i \in I} T_{i}\right): X \times Y \rightarrow 2^{X \times Y}$ defined as $(D \times T)(x, y)=\prod_{i \in I} D_{i}(x, y) \times \prod_{i \in I} T_{i}(x, y)$, $\forall(x, y) \in X \times Y$, is $\Phi$-condensing and for each $i \in I$, the conditions (i), (ii) and (iii) of Corollary 3.4 hold. Then the solution set of the (SGQEP) is nonempty.

Remark 3.6. Theorem 3.3 is a generalization of [19, Theorem 3]. Corollaries 3.4 and 3.5 extend and generalize the main results in [10-17].

\section{Applications}

In this section, we present some existence of a solution for the (G-Debreu VEP) and the (GDebreu EP).

Theorem 4.1. Let $I$ be any index set. For each $i \in I$, let $Z_{i}$ be a topological vector space, let $E_{i}$ and $F_{i}$ be two Hausdorff topological vector spaces, let $X_{i} \subseteq E_{i}$ and $Y_{i} \subseteq F_{i}$ be nonempty and convex subsets, let $C_{i}: X \rightarrow 2^{Z_{i}}$ be a set-valued map such that $C_{i}(x)$ is a proper, closed and convex cone with apex at the origin and int $C_{i}(x) \neq \emptyset$ for each $x \in X$ and $P_{i}=\cap_{x \in X} C_{i}(x), D_{i}: X \times Y \rightarrow 2^{X_{i}}$ and $T_{i}: X \times Y \rightarrow 2^{Y_{i}}$ be set-valued maps with nonempty convex values and open lower sections, the set $W_{i}=\left\{(x, y) \in X \times Y: x_{i} \in D_{i}(x, y)\right.$ and $\left.y_{i} \in T_{i}(x, y)\right\}$ be closed in $X \times Y$ and $\phi_{i}$ be a bifunction 
from $X \times Y$ into $Z_{i}$. For each $i \in I$, assume that

(i) $M_{i}=Z_{i} \backslash\left(-\operatorname{int} C_{i}\right): X \rightarrow 2^{Z_{i}}$ is upper semicontinuous;

(ii) For all $x^{i} \in X^{i}$ and $y \in Y, z_{i} \mapsto \phi_{i}\left(x^{i}, y, z_{i}\right)$ is natural $P_{i}$-quasifunction, where $P_{i}=$ $\cap_{x \in X} C_{i}(x)$;

(iii) $\phi_{i}$ is continuous on $X \times Y$;

(iv) there exist nonempty and compact subsets $N \subseteq X$ and $K \subseteq Y$ and nonempty, compact and convex subsets $B_{i} \subseteq X_{i}, A_{i} \subseteq Y_{i}$ for each $i \in I$ such that $\forall(x, y)=\left(x^{i}, x_{i}, y\right) \in$ $X \times Y \backslash N \times K \exists i \in I$ and $\exists \bar{u}_{i} \in B_{i}, \bar{v}_{i} \in A_{i}$ satisfying $\bar{u}_{i} \in D_{i}(x, y), \bar{v}_{i} \in T_{i}(x, y)$ and $\phi_{i}\left(x^{i}, y, \bar{u}_{i}\right)-\phi_{i}(x, y) \in-\operatorname{int} C_{i}(x)$.

Then, there exists $(\bar{x}, \bar{y})=\left(\bar{x}^{i}, \bar{x}_{i}, \bar{y}^{i}, \bar{y}_{i}\right)$ in $X \times Y$ such that for each $i \in I$,

$$
\bar{x}_{i} \in D_{i}(\bar{x}, \bar{y}), \quad \bar{y}_{i} \in T_{i}(\bar{x}, \bar{y}): \phi_{i}\left(\bar{x}^{i}, \bar{y}, z_{i}\right)-\phi_{i}(\bar{x}, \bar{y}) \notin-\operatorname{int} C_{i}(\bar{x}), \quad \forall z_{i} \in D_{i}(\bar{x}, \bar{y}) .
$$

That is, the solution set of the (G-Debreu VEP) is nonempty.

Proof. For each $i \in I$, we define a trifunction $f_{i}: X \times Y \times X_{i}$ as

$$
f_{i}\left(x, y, u_{i}\right)=\phi_{i}\left(x^{i}, y, u_{i}\right)-\phi_{i}(x, y), \quad \forall\left(x, y, u_{i}\right) \in X \times Y \times X_{i}
$$

Since $\phi_{i}\left(x^{i}, y, \cdot\right)$ is natural $P_{i}$ quasi-function, by [19, Remark 2], for all $u_{i_{1}}, u_{i_{2}} \in X_{i}$ and for all $\lambda \in[0,1], \exists \alpha \in[0,1]$ such that

$$
\phi_{i}\left(x^{i}, y, \lambda u_{i_{1}}+(1-\lambda) u_{i_{2}}\right) \in \alpha \phi_{i}\left(x^{i}, y, u_{i_{1}}\right)+(1-\alpha) \phi_{i}\left(x^{i}, y, u_{i_{2}}\right)-P_{i}
$$

Hence

$$
f_{i}\left(x, y, \lambda u_{i_{1}}+(1-\lambda) u_{i_{2}}\right) \in \alpha f_{i}\left(x, y, u_{i_{1}}\right)+(1-\alpha) f_{i}\left(x, y, u_{i_{2}}\right)-P_{i} .
$$

Hence, for all $(x, y) \in X \times Y, f_{i}(x, y, \cdot)$ is natural $P_{i}$ quasifunction.

By condition (iii), we know that for all $z_{i} \in X_{i}$, the map $(x, y) \mapsto f_{i}\left(x, y, z_{i}\right)$ is continuous on $X \times Y$. So it follows from Remark 3.2 that condition (iii) of Theorem 3.1 holds. It is easy to verify that the other conditions of Theorem 3.1 are satisfied. By Theorem 3.1, we know that the conclusion holds. results.

Similarly, by Theorem 3.3, Corollaries 3.4 and 3.5, respectively, we have the following

Theorem 4.2. Let $I$ be any index set. For each $i \in I$, let $Z_{i}$ be a topological vector space, let $E_{i}$ and $F_{i}$ be two locally convex Hausdorff topological vector spaces, let $X_{i} \subseteq E_{i}$ and $Y_{i} \subseteq F_{i}$ be nonempty, closed and convex subsets, let $C_{i}: X \rightarrow 2^{Z_{i}}$ be a set-valued map such that $C_{i}(x)$ is a proper, closed and convex cone with apex at the origin and int $C_{i}(x) \neq \emptyset$ for each $x \in X$ and $P_{i}=\cap_{x \in X} C_{i}(x)$, $D_{i}: X \times Y \rightarrow 2^{X_{i}}$ and $T_{i}: X \times Y \rightarrow 2^{Y_{i}}$ be set-valued maps with nonempty convex values and open lower sections, the set $W_{i}=\left\{(x, y) \in X \times Y: x_{i} \in D_{i}(x, y)\right.$ and $\left.y_{i} \in T_{i}(x, y)\right\}$ be 
closed in $X \times Y$ and $\varphi_{i}: X \times Y \rightarrow Z_{i}$ be a vector-valued function. Assume that the set-valued map $D \times T=\left(\prod_{i \in I} D_{i} \times \prod_{i \in I} T_{i}\right): X \times Y \rightarrow 2^{X \times Y}$ defined as $(D \times T)(x, y)=\prod_{i \in I} D_{i}(x, y) \times \prod_{i \in I} T_{i}(x, y)$, $\forall(x, y) \in X \times Y$, is $\Phi$-condensing and (i), (ii), and (iii) of Theorem 4.1 hold. Then, the solution set of the (G-Debreu VEP) is nonempty.

Theorem 4.3. Let $I$ be any index set. For each $i \in I$, let $X_{i} \subseteq E_{i}$ and $Y_{i} \subseteq F_{i}$ be nonempty and convex subsets, let $D_{i}: X \times Y \rightarrow 2^{X_{i}}$ and $T_{i}: X \times Y \rightarrow 2^{Y_{i}}$ be set-valued maps with nonempty convex values and open lower sections, the set $W_{i}=\left\{(x, y) \in X \times Y: x_{i} \in D_{i}(x, y)\right.$ and $\left.y_{i} \in T_{i}(x, y)\right\}$ be closed in $X \times Y$ and $\phi_{i}$ be a bifunction from $X \times Y$ into $R$. For each $i \in I$, assume that

(i) for all $x^{i} \in X^{i}$ and $y \in Y, z_{i} \mapsto \phi_{i}\left(x^{i}, y, z_{i}\right)$ is quasiconvex;

(ii) $\phi_{i}$ is continuous on $X \times Y$;

(iii) there exist nonempty and compact subsets $N \subseteq X$ and $K \subseteq Y$ and nonempty, compact and convex subsets $B_{i} \subseteq X_{i}, A_{i} \subseteq Y_{i}$ for each $i \in I$ such that $\forall(x, y)=\left(x^{i}, x_{i}, y\right) \in$ $X \times Y \backslash N \times K \exists i \in I$ and $\exists \bar{u}_{i} \in B_{i}, \bar{v}_{i} \in A_{i}$ satisfying $\bar{u}_{i} \in D_{i}(x, y), \bar{v}_{i} \in T_{i}(x, y)$ and $\phi_{i}\left(x^{i}, y, \bar{u}_{i}\right)<\phi_{i}(x, y)$.

Then, there exists $(\bar{x}, \bar{y})=\left(\bar{x}^{i}, \bar{x}_{i}, \bar{y}^{i}, \bar{y}_{i}\right)$ in $X \times Y$ such that for each $i \in I$,

$$
\bar{x}_{i} \in D_{i}(\bar{x}, \bar{y}), \quad \bar{y}_{i} \in T_{i}(\bar{x}, \bar{y}): \phi_{i}\left(\bar{x}^{i}, \bar{y}, z_{i}\right) \geq \phi_{i}(\bar{x}, \bar{y}), \quad \forall z_{i} \in D_{i}(\bar{x}, \bar{y}) .
$$

That is, the solution set of the (G-Debreu EP) is nonempty.

Theorem 4.4. Let $I$ be any index set. For each $i \in I$, let $E_{i}$ and $F_{i}$ be two locally convex Hausdorff topological vector spaces, $X_{i} \subseteq E_{i}$ and $Y_{i} \subseteq F_{i}$ be nonempty, closed and convex subsets, let $D_{i}$ : $X \times Y \rightarrow 2^{X_{i}}$ and $T_{i}: X \times Y \rightarrow 2^{Y_{i}}$ be set-valued maps with nonempty convex values and open lower sections, the set $W_{i}=\left\{(x, y) \in X \times Y: x_{i} \in D_{i}(x, y)\right.$ and $\left.y_{i} \in T_{i}(x, y)\right\}$ be closed in $X \times Y$ and $\varphi_{i}: X \times Y \rightarrow R$ be a function. Assume that the set-valued map $D \times T=\left(\prod_{i \in I} D_{i} \times \prod_{i \in I} T_{i}\right): X \times Y \rightarrow$ $2^{X \times Y}$ defined as $(D \times T)(x, y)=\prod_{i \in I} D_{i}(x, y) \times \prod_{i \in I} T_{i}(x, y), \forall(x, y) \in X \times Y$, is $\Phi$-condensing and (i), and (ii) of Theorem 4.3 hold. Then, the solution set of the (G-Debreu EP) is nonempty.

Remark 4.5. Theorem 4.1 extends and generalizes [19, Theorem 5] and [20, Theorems 3.1, 3.6 and Corollaries 3.2, 3.3, and 3.5]. Theorem 4.2 extends and generalizes [19, Theorem 6]. Theorems 4.3 and 4.4 are generalizations of [20, Corollaries 3.5 and 3.7] and the corresponding results in [21-24].

\section{Acknowledgment}

This research was supported by the National Natural Science Foundation of China (Grant no. 10771228 and Grant no. 10831009).

\section{References}

[1] F. Giannessi, Vector Variational Inequalities and Vector Equilibria. Mathematical Theories, vol. 38 of Nonconvex Optimization and Its Applications, Kluwer Academic Publishers, Dordrecht, The Netherlands, 2000.

[2] X. H. Gong, "Efficiency and Henig efficiency for vector equilibrium problems," Journal of Optimization Theory and Applications, vol. 108, no. 1, pp. 139-154, 2001. 
[3] M. Bianchi, N. Hadjisavvas, and S. Schaible, "Vector equilibrium problems with generalized monotone bifunctions," Journal of Optimization Theory and Applications, vol. 92, no. 3, pp. 527-542, 1997.

[4] Q. H. Ansari, X. Q. Yang, and J.-C. Yao, "Existence and duality of implicit vector variational problems," Numerical Functional Analysis and Optimization, vol. 22, no. 7-8, pp. 815-829, 2001.

[5] W. Oettli, "A remark on vector-valued equilibria and generalized monotonicity," Acta Mathematica Vietnamica, vol. 22, no. 1, pp. 213-221, 1997.

[6] N. Hadjisavvas and S. Schaible, "From scalar to vector equilibrium problems in the quasimonotone case," Journal of Optimization Theory and Applications, vol. 96, no. 2, pp. 297-309, 1998.

[7] N. X. Tan and P. N. Tinh, "On the existence of equilibrium points of vector functions," Numerical Functional Analysis and Optimization, vol. 19, no. 1-2, pp. 141-156, 1998.

[8] Q. H. Ansari and J. C. Yao, "On vector quasi-quasi-equilibrium problems," in Equilibrium Problems and Variational Models, P. Daniele, F. Giannessi, and A. Maugeri, Eds., Kluwer Academic Publishers, Dordrecht, The Netherlands, 2002.

[9] Y. Chiang, O. Chadli, and J. C. Yao, "Existence of solutions to implicit vector variational inequalities," Journal of Optimization Theory and Applications, vol. 116, no. 2, pp. 251-264, 2003.

[10] J. W. Peng, "Quasi-equilibrium problem on W-spase," Journal of Chongqiong Normal University, vol. 17, no. 4, pp. 36-40, 2000 (Chinese).

[11] P. Cubiotti, "Existence of Nash equilibria for generalized games without upper semicontinuity," International Journal of Game Theory, vol. 26, no. 2, pp. 267-273, 1997.

[12] J. Zhou and G. Chen, "Diagonal convexity conditions for problems in convex analysis and quasivariational inequalities," Journal of Mathematical Analysis and Applications, vol. 132, no. 1, pp. 213-225, 1988.

[13] J.-P. Aubin and I. Ekeland, Applied Nonlinear Analysis, Pure and Applied Mathematics, John Wiley \& Sons, New York, NY, USA, 1984.

[14] S. Chang, B. S. Lee, X. Wu, Y. J. Cho, and G. M. Lee, “On the generalized quasi-variational inequality problems," Journal of Mathematical Analysis and Applications, vol. 203, no. 3, pp. 686-711, 1996.

[15] M.-P. Chen, L.-J. Lin, and S. Park, "Remarks on generalized quasi-equilibrium problems," Nonlinear Analysis: Theory, Methods \& Applications, vol. 52, no. 2, pp. 433-444, 2003.

[16] L.-J. Lin and Z.-T. Yu, "Fixed points theorems of KKM-type maps," Nonlinear Analysis: Theory, Methods $\mathcal{E}$ Applications, vol. 38, no. 2, pp. 265-275, 1999.

[17] L.-J. Lin and S. Park, "On some generalized quasi-equilibrium problems," Journal of Mathematical Analysis and Applications, vol. 224, no. 2, pp. 167-181, 1998.

[18] Q. H. Ansari, S. Schaible, and J. C. Yao, "System of vector equilibrium problems and its applications," Journal of Optimization Theory and Applications, vol. 107, no. 3, pp. 547-557, 2000.

[19] Q. H. Ansari, W. K. Chan, and X. Q. Yang, "The system of vector quasi-equilibrium problems with applications," Journal of Global Optimization, vol. 29, no. 1, pp. 45-57, 2004.

[20] J. W. Peng, X. M. Yang, and D. L. Zhu, "System of vector quasi-equilibrium problems and its applications," Applied Mathematics and Mechanics, vol. 27, no. 8, pp. 1107-1114, 2006.

[21] C. Ionescu Tulcea, "On the approximation of upper semi-continuous correspondences and the equilibriums of generalized games," Journal of Mathematical Analysis and Applications, vol. 136, no. 1, pp. 267-289, 1988

[22] G. X.-Z. Yuan, G. Isac, K.-K. Tan, and J. Yu, “The study of minimax inequalities, abstract economics and applications to variational inequalities and Nash equilibria," Acta Applicandae Mathematicae, vol. 54, no. 2, pp. 135-166, 1998.

[23] W. Shafer and H. Sonnenschein, "Equilibrium in abstract economies without ordered preferences," Journal of Mathematical Economics, vol. 2, no. 3, pp. 345-348, 1975.

[24] J. Nash, "Non-cooperative games," Annals of Mathematics, vol. 54, pp. 286-295, 1951.

[25] D. T. Luc, Theory of Vector Optimization, vol. 319 of Lecture Notes in Economics and Mathematical Systems, Springer, Berlin, Germany, 1989.

[26] P. M. Fitzpatrick and W. V. Petryshyn, "Fixed point theorems for multivalued noncompact acyclic mappings," Pacific Journal of Mathematics, vol. 54, no. 2, pp. 17-23, 1974.

[27] P. Deguire, K. K. Tan, and G. X.-Z. Yuan, "The study of maximal elements, fixed points for $L_{S^{-}}$ majorized mappings and their applications to minimax and variational inequalities in product topological spaces," Nonlinear Analysis: Theory, Methods \& Applications, vol. 37, no. 7, pp. 933-951, 1999.

[28] S. Chebbi and M. Florenzano, "Maximal elements and equilibria for condensing correspondences," Nonlinear Analysis: Theory, Methods \& Applications, vol. 38, no. 8, pp. 995-1002, 1999. 


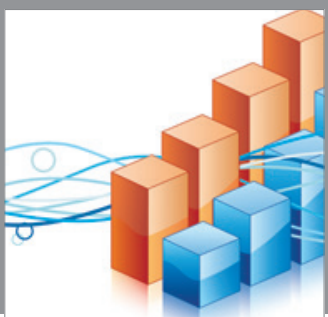

Advances in

Operations Research

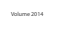

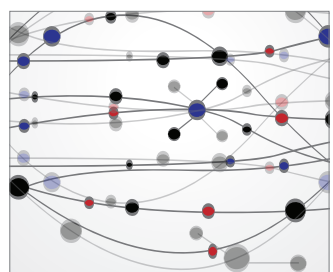

\section{The Scientific} World Journal
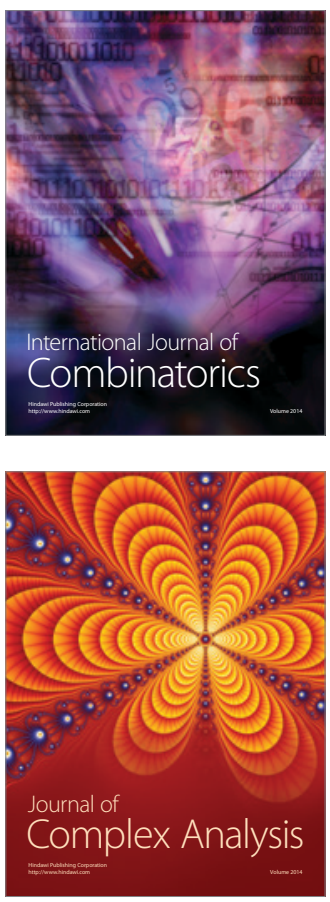

International Journal of

Mathematics and

Mathematical

Sciences
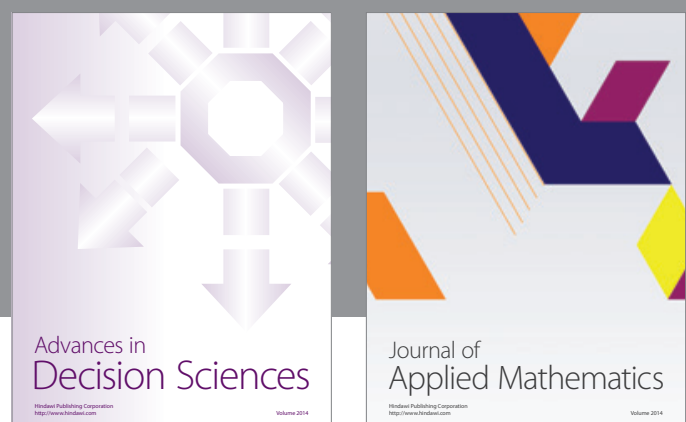

Journal of

Applied Mathematics
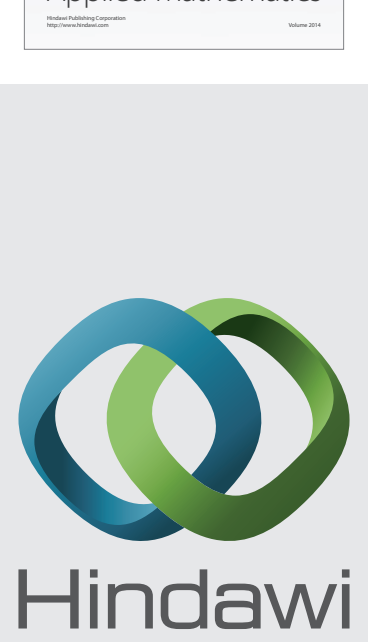

Submit your manuscripts at http://www.hindawi.com
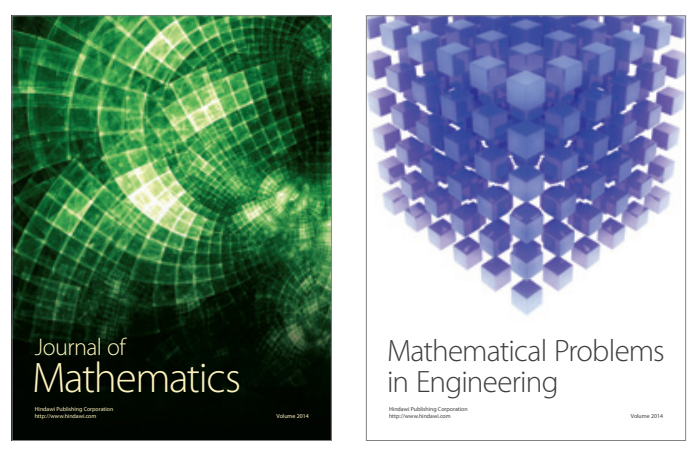

Mathematical Problems in Engineering
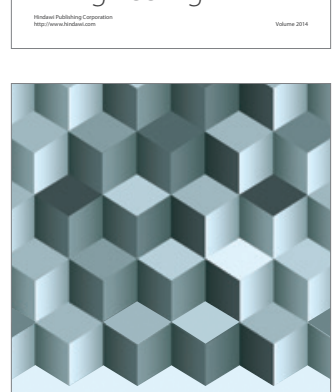

Journal of

Function Spaces
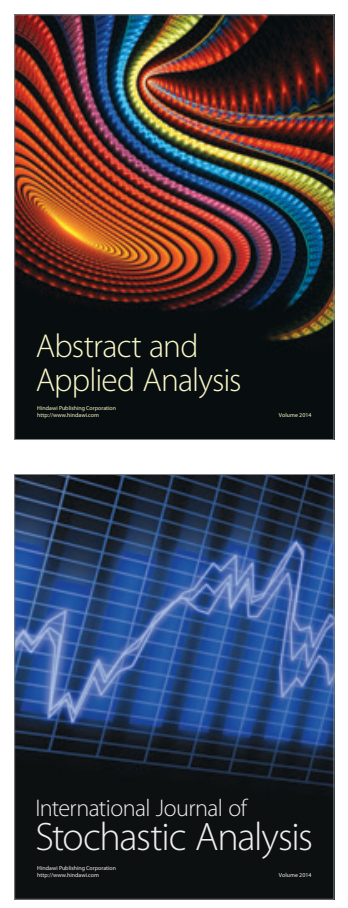

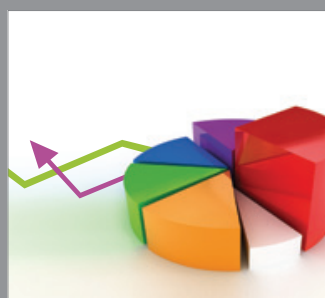

ournal of

Probability and Statistics

Promensencen
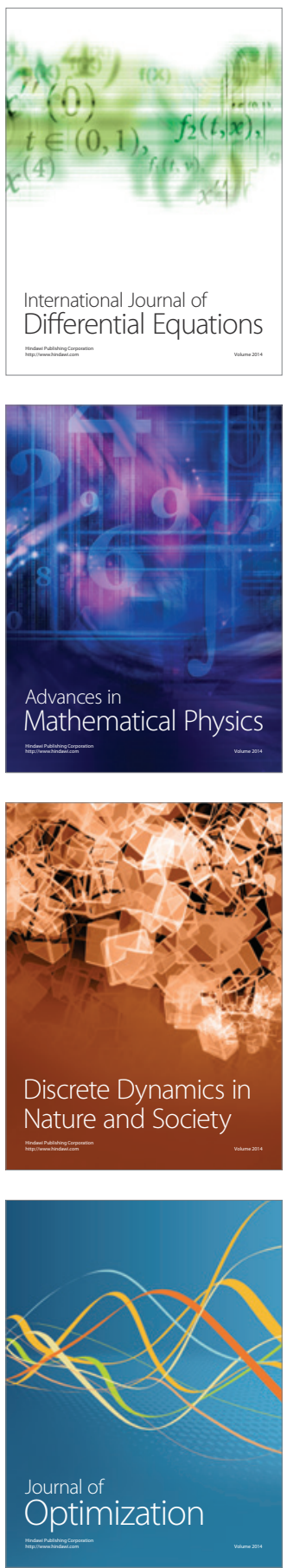